\title{
Toward criteria for pragmatic measurement in implementation research and practice: a stakeholder-driven approach using concept mapping
}

\author{
Byron J. Powell ${ }^{1 *}$, Cameo F. Stanick², Heather M. Halko ${ }^{3}$, Caitlin N. Dorsey ${ }^{4}$, Bryan J. Weiner ${ }^{5}$, Melanie A. Barwick ${ }^{6}$, \\ Laura J. Damschroder ${ }^{7}$, Michel Wensing ${ }^{8}$, Luke Wolfenden ${ }^{9}$ and Cara C. Lewis ${ }^{4}$
}

\begin{abstract}
Background: Advancing implementation research and practice requires valid and reliable measures of implementation determinants, mechanisms, processes, strategies, and outcomes. However, researchers and implementation stakeholders are unlikely to use measures if they are not also pragmatic. The purpose of this study was to establish a stakeholder-driven conceptualization of the domains that comprise the pragmatic measure construct. It built upon a systematic review of the literature and semi-structured stakeholder interviews that generated 47 criteria for pragmatic measures, and aimed to further refine that set of criteria by identifying conceptually distinct categories of the pragmatic measure construct and providing quantitative ratings of the criteria's clarity and importance.

Methods: Twenty-four stakeholders with expertise in implementation practice completed a concept mapping activity wherein they organized the initial list of 47 criteria into conceptually distinct categories and rated their clarity and importance. Multidimensional scaling, hierarchical cluster analysis, and descriptive statistics were used to analyze the data.

Findings: The 47 criteria were meaningfully grouped into four distinct categories: (1) acceptable, (2) compatible, (3) easy, and (4) useful. Average ratings of clarity and importance at the category and individual criteria level will be presented.

Conclusions: This study advances the field of implementation science and practice by providing clear and conceptually distinct domains of the pragmatic measure construct. Next steps will include a Delphi process to develop consensus on the most important criteria and the development of quantifiable pragmatic rating criteria that can be used to assess measures.
\end{abstract}

\section{Background}

Bridging the gap between research and practice by advancing implementation science will require valid and reliable measures of implementation determinants, mechanisms, processes, strategies, and outcomes [1]. However, implementation stakeholders (i.e., researchers and practice-based implementers) are unlikely to use measures solely on the basis of strong psychometric

\footnotetext{
* Correspondence: bjpowell@unc.edu

${ }^{1}$ Department of Health Policy and Management, Gillings School of Global Public Health, University of North Carolina at Chapel Hill, $1105 \mathrm{C}$

McGavran-Greenberg Hall, 135 Dauer Drive, Campus Box 7411, Chapel Hill, NC 27599, USA

Full list of author information is available at the end of the article
}

properties; they also need to be pragmatic [2, 3]. For example, a measure that is psychometrically sound, but is time-consuming or expensive to administer, is unlikely to be used. There is currently no consensus about what constitutes a pragmatic measure. Glasgow and Riley [2] advanced the conceptualization of the pragmatic measure construct by suggesting two types of criteria: required (important to stakeholders, low burden for respondents and staff, actionable, and sensitive to change) and recommended (broadly applicable, used for benchmarking, unlikely to cause harm, psychometrically strong, and related to theory or model). However, these recommendations may be limited as they were not 
developed through a systematic literature review, were not informed by relevant stakeholders, and focused on clinical measures. Key aspects of the pragmatic measure construct may have been overlooked.

The "Advancing Implementation Science through Measure Development and Evaluation" study [3] aims to (1) establish a stakeholder-driven operationalization of pragmatic measures and develop reliable, valid rating criteria for assessing this construct; (2) develop reliable, valid, and pragmatic measures of three different implementation outcomes [4] (acceptability, appropriateness, and feasibility) [5]; and (3) identify measures that demonstrate both psychometric and pragmatic strength. This article details our Aim 1 efforts to establish a stakeholder-driven conceptualization of the domains that comprise the pragmatic measure construct. As a first step toward that aim, we conducted a systematic review of the literature and semi-structured interviews with stakeholders drawn from multiple organization types (e.g., community mental health center, schoolbased mental health, state mental health department, residential treatment center, and inpatient hospital) and service roles (e.g., administrators and clinicians). The eight relevant articles from the systematic review and the seven semi-structured interviews ultimately yielded 47 potential criteria for pragmatic measures (e.g., low cost, efficient, easy to score) after duplicates were removed [6].

The present study engaged stakeholders with experience implementing behavioral health interventions in a concept mapping activity [7] to explore the relationships between the criteria, to develop conceptually distinct categories, and to assess the clarity and importance of the criteria. Considering this list of criteria and the associated ratings of clarity and importance will help implementation stakeholders to develop or select measures that are pragmatic. The findings of this study will assist us in refining and consolidating the list of criteria as we work toward developing a valid and reliable set of rating criteria for assessing the extent to which a measure is pragmatic. Ultimately, the rating criteria will be applied to implementation-related measures of constructs associated with the Consolidated Framework for Implementation Research [8] and the Implementation Outcomes Framework [4] in Aim 3 of our study [3].

\section{Methods}

\section{Participants}

Purposeful sampling [9] was used to recruit stakeholders $(N=24)$ with experience implementing behavioral health interventions and to ensure maximum variation in discipline, setting, and geographic location. The stakeholders were administrators $(n=13)$, clinicians $(n=6)$, and researchers $(n=5)$ with an average of $10(\mathrm{SD}=9)$ years of implementation experience. They worked in community mental health $(n=10)$; specialty mental health, outpatient mental health, or private practice $(n=3)$; community organizations $(n=3)$; primary care $(n=2)$; children's social services $(n=1)$; inpatient psychiatry $(n=1)$; schools $(n=1)$; government agencies $(n=1)$; and other settings $(n=2)$. Twenty-four stakeholders are above the recommended sample size for concept mapping $(\geq 15)$ [10].

\section{Data collection}

Stakeholders completed a concept mapping activity, which is a structured process designed to organize concepts into categories and generate ratings of specified dimensions $[7,11,12]$. It is particularly useful for structuring the ideas of diverse groups of stakeholders and has been used in implementation research for multiple purposes, including identifying and prioritizing implementation barriers and facilitators [13, 14], organizing implementation strategies [15], and identifying training needs [16]. Concept mapping is an inherently mixed methods approach that involves multiple steps, typically including brainstorming, statement analysis and synthesis, unstructured sorting of statements, multidimensional scaling and cluster analysis, and the generation of interpretable maps and data displays [7]. Thorough and accessible introductions to the concept mapping method can be found in Trochim and Kane [11] and Kane and Trochim [7].

The criteria for pragmatic measures were generated through a systematic review of the literature and semistructured interviews with stakeholders (described above) that yielded 47 criteria after duplicates were removed [6]. The Concept Systems Global MAX ${ }^{\mathrm{sm}}$ [17] web-based platform was used to collect and analyze the data for this study asynchronously. After logging on to the web-based platform, participants were asked to complete basic demographic questions (primary role, work setting, years of experience, and race/ethnicity). They were then asked to complete an unstructured sorting task that involved sorting each of the 47 criteria into conceptually similar groups and giving each category a name that describes its theme or contents. They were instructed not to sort based upon priority or value (e.g., "important" or "hard to do") or to create "miscellaneous" or "other" piles that grouped dissimilar items. It was also noted that the number of categories participants create typically varies from 5 to 20 ; however, there was no mandate to stay within that range. To help us to determine the criteria that may be most helpful as we move toward developing concrete rating scales for the pragmatic construct, we asked stakeholders to rate each criterion's clarity and importance on a 10-point scale $(1=$ not at all clear/not at all important, $10=$ incredibly clear/incredibly important). Data collection was completed in 2 months. 


\section{Data analysis}

Through the Concept Systems Global MAX ${ }^{\mathrm{TM}}$ [17] webbased platform, multidimensional scaling and hierarchical cluster analysis were used to generate visualizations of the relationships between the pragmatic criteria. Multidimensional scaling was used to generate a point map depicting each of the pragmatic criteria and relationships between them based upon a summed square similarity matrix [11]. Criteria frequently sorted together were placed closer together on the point map. Hierarchical cluster analysis was used to partition the point map into non-overlapping clusters [11].

The analytic process involved the investigative team considering a range of cluster solutions, deciding which solution best suited the purposes of the current study, and labeling each cluster [7, 11]. Concept Systems Global $\mathrm{MAX}^{\mathrm{Tm}}$ [17] aids in the labeling process by suggesting potential cluster labels based upon participant responses. These labels do not always adequately reflect the items within a cluster; however, in at least one case, we used a variant of the suggested label, and in others, the suggested labels inspired us to generate labels that had similar meanings as we sought to obtain consensus among the investigative team. In two cases, individual items were moved from one cluster to another to improve the clarity and consistency of the clusters [7]. Model fit was assessed using the stress value, an indicator of goodness of fit between the point map and the total similarity matrix. Cross-study syntheses of concept mapping studies have consistently found mean stress values of $0.28[7,10,12]$, with higher stress scores indicating poorer representation of the data. The final cluster solution and associated labels were vetted by a stakeholder panel that included four of the seven individuals (three did not respond) from the semi-structured interview study [6] and by all nine members of the parent study's International Advisory Board, which is comprised of leading implementation scientists purposefully selected to represent broad expertise and geographic diversity. The four stakeholders participated in both the interview study and the concept mapping exercise, while members of the International Advisory Board were not involved in either study as participants.

Descriptive statistics were used to characterize clarity and importance ratings at both the criterion and cluster levels. At the criterion level, means for both clarity and importance are captured in Table 1 and in Fig. 3 which depicts a "go zone" graph $[7,11]$. The go zone graph is a bivariate plot of the clarity and importance ratings that is divided into quadrants based upon the mean values of those dimensions. Thus, criteria that fall into quadrant I are above the mean for both importance and clarity, whereas criteria that fall into quadrant III are below the mean for both importance and clarity. The go zone quadrants for each criterion are listed in Table 1 as well to ease the interpretation of each criterion's mean ratings. Finally, the overall mean for importance and clarity was calculated for each cluster and is shown in a ladder graph (or pattern match) [7, 11] in Fig. 2.

\section{Results}

Valid sorts were obtained from 23 stakeholders. One stakeholder sorted the criteria into value-based categories (e.g., "not that important") and was dropped from the multidimensional scaling and hierarchical cluster analyses. All 24 stakeholders provided valid ratings of clarity and importance.

The investigative team independently considered cluster solutions ranging from ten to two clusters and came to consensus on a four-cluster solution with the following labels: acceptable, compatible, easy, and useful. The stakeholder panel $(n=4)$ and an International Advisory Board $(n=9)$ vetted the four-cluster solution and agreed that it balanced parsimony and distinction between the domains. The stress value was 0.2581 , demonstrating goodness of fit $[7,10,12]$. Figure 1 shows the final point and cluster map that visually represents the relationships between the 47 criteria. Each point in Fig. 1 represents a single criterion, which are labeled numerically to facilitate cross-referencing the point and cluster map with the full descriptions listed in Table 1. Two criteria ("important to clinical care" [\#7] and "sensitive to change" [\#19]) were moved from the "Compatible" cluster to the "Useful" cluster to enhance conceptual clarity and consistency.

Figure 2 shows the mean clarity and importance ratings at the cluster level. Overall, the mean ratings for the clusters were relatively high for both clarity (7.06-7.86) and importance (7.16-8.06). Figure 3 depicts a "go-zone" graph that shows clarity and importance ratings for each of the 47 criteria and where each criterion falls with respect to the overall mean rating for clarity (7.6) and importance (7.52).

\section{Discussion}

To usefully inform the assessment of implementation determinants, mechanisms, processes, strategies, and outcomes, measures must be both psychometrically sound and pragmatic. This study advances previous work [2, 6] by engaging stakeholders to conceptualize domains that comprise the pragmatic measure construct. The 47 criteria previously identified through a systematic literature review and semi-structured interviews [6] were grouped into four categories: acceptable, compatible, easy, and useful. The overarching categories should be helpful in considering the pragmatic construct and have the advantage of parsimony. However, at this stage of development, we suggest that readers consider the 
Table 1 Mean clarity and importance ratings for each criterion $(n=24)$

\begin{tabular}{|c|c|c|c|c|}
\hline \# & Criterion & Clarity & Importance & Quad. \\
\hline \multicolumn{5}{|c|}{ Acceptable } \\
\hline 4 & Creates a low social desirability bias & 5.21 & 5.88 & III \\
\hline 22 & Transparent & 6.75 & 6.92 & III \\
\hline 24 & Acceptable (to staff and clients) & 7.83 & 8.50 & 1 \\
\hline 25 & Tied to reimbursement & 8.00 & 5.08 & $\|$ \\
\hline 28 & Relevant & 7.21 & 8.71 & IV \\
\hline 30 & Offers relative advantage over ex & 7.33 & 7.54 & IV \\
\hline 43 & Low cost & 8.67 & 8.04 & 1 \\
\hline \multicolumn{5}{|c|}{ Compatible } \\
\hline 3 & Applicable & 7.25 & 8.25 & IV \\
\hline 8 & Efficient & 7.79 & 8.21 & I \\
\hline 12 & Focused & 5.92 & 6.92 & III \\
\hline 16 & The output of routine activities & 6.58 & 7.21 & III \\
\hline 37 & Not used for staff punishment & 7.63 & 7.63 & । \\
\hline 40 & Non-duplicative & 7.21 & 7.50 & III \\
\hline \multicolumn{5}{|l|}{ Easy } \\
\hline 9 & Offers flexible administration time & 6.88 & 6.92 & III \\
\hline 10 & Easy to interpret & 8.88 & 8.38 & I \\
\hline 15 & Creates low assessor burden (ease of training, scoring, administration time) & 8.50 & 7.75 & 1 \\
\hline 17 & Easy to administer & 8.75 & 8.13 & 1 \\
\hline 20 & Not wordy & 8.79 & 6.38 & $\|$ \\
\hline 21 & Completed with ease & 8.75 & 7.71 & I \\
\hline 23 & Requires no expertise & 7.46 & 4.75 & III \\
\hline 26 & Of low complexity & 7.58 & 6.42 & III \\
\hline 27 & Uses accessible language & 7.75 & 8.13 & I \\
\hline 31 & Accessible by phone & 8.29 & 4.88 & $\|$ \\
\hline 32 & Brief & 8.21 & 6.92 & $\|$ \\
\hline 34 & Intuitive & 6.29 & 6.25 & III \\
\hline 36 & Feasible & 7.00 & 8.25 & IV \\
\hline 39 & Simple & 7.54 & 7.17 & III \\
\hline 41 & Easy to use & 8.29 & 8.00 & 1 \\
\hline 42 & Easy to score & 8.88 & 7.75 & I \\
\hline 44 & One that offers automated scoring or can be scored elsewhere & 8.63 & 6.71 & $\|$ \\
\hline 45 & Offers a compatible format to setting/user & 5.63 & 7.29 & III \\
\hline 47 & Low burden & 7.33 & 8.21 & IV \\
\hline \multicolumn{5}{|c|}{ Useful } \\
\hline 1 & Informs decision making & 8.00 & 8.71 & I \\
\hline 2 & Fits organizational activities & 8.21 & 7.96 & I \\
\hline 5 & Provides a cut-off score leading to an intervention or treatment plan & 7.63 & 6.96 & $\|$ \\
\hline 6 & Connects to clinical outcomes & 8.38 & 8.83 & । \\
\hline 7 & Important to clinical care & 7.96 & 8.92 & 1 \\
\hline 11 & Produces reliable and valid results & 9.13 & 9.25 & । \\
\hline 13 & Reveals problems/issues in process or outcomes & 6.79 & 6.67 & IV \\
\hline 14 & Informs adherence of fidelity & 7.54 & 7.42 & III \\
\hline
\end{tabular}


Table 1 Mean clarity and importance ratings for each criterion $(n=24)$ (Continued)

\begin{tabular}{|c|c|c|c|c|}
\hline \# & Criterion & Clarity & Importance & Quad. \\
\hline 18 & Assesses organizational progress over time & 7.50 & 7.67 & IV \\
\hline 19 & Sensitive to change & 7.25 & 7.25 & III \\
\hline 29 & Meaningful & 6.79 & 8.71 & IV \\
\hline 33 & Confirms efficacy of interventions & 7.13 & 7.92 & IV \\
\hline 35 & Has a meaningful score distribution & 6.54 & 6.71 & III \\
\hline 38 & Optimizes patient care & 7.46 & 8.83 & IV \\
\hline 46 & Informs clinical intervention selection & 7.92 & 8.29 & 1 \\
\hline
\end{tabular}

nuances of the pragmatic construct that are represented at the criterion level.

Ratings of clarity and importance at criterion and cluster levels were generally high. Implementation stakeholders interested in using these criteria to inform the development or assessment of measures may wish to focus on the criteria that fell within the go zone (i.e., above the overall mean for both importance and clarity), as those criteria are likely closer to being useable in their current form. Ratings for some of the other criteria suggested items that need to be removed due to lack of importance (e.g., "requires no expertise,") or edited due to lack of clarity (e.g., "focused").

This study is a step toward developing rating criteria that could inform measure development and the assessment of measures' pragmatic qualities, which ultimately will benefit research and practice by yielding and revealing measures that are psychometrically strong and pragmatic, possibly increasing their future use. Next steps will include developing consensus on the relative priorities for these categories and criteria through a Delphi [18] study; developing rating criteria with concrete, measurable anchors; and assessing inter-rater reliability and known-groups validity of the criteria [3]. Longer-term objectives are to combine the pragmatic rating criteria with evidence-based rating criteria and apply both to a repository of over 450 measures to assess their psychometric and pragmatic strength $[3,19]$. The resulting pragmatic rating scale may also influence reporting guidelines for implementation measures and measure development procedures.

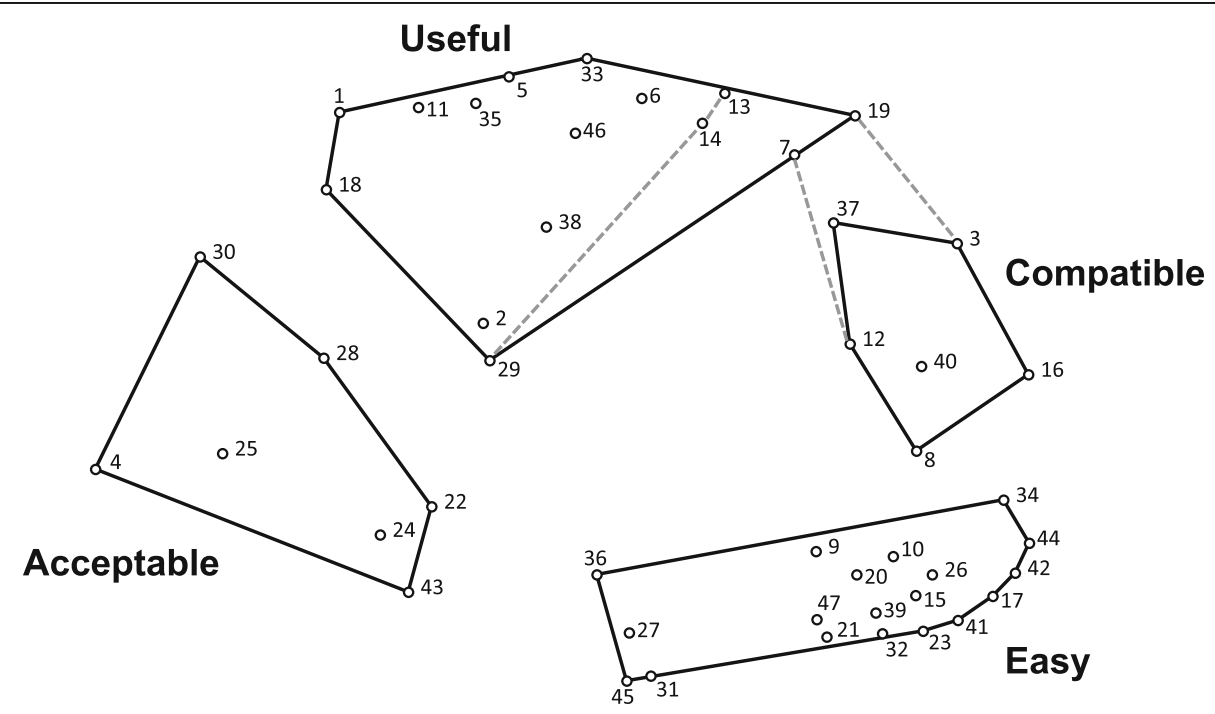

Fig. 1 Point and cluster map of criteria demonstrating spatial relationships $(n=23)$. This point and cluster map reflects the product of our stakeholders' (valid response $n=23$ ) sorting the 47 criteria into groups that they deemed conceptually similar. Each strategy is depicted as a dot with a number that corresponds to Table 1. The distances between criteria reflect the frequency at which they were sorted together; thus, strategies that were sorted together frequently are closer together on the map. These spatial relationships are relative to the data in this study and do not reflect an absolute relationship (i.e., a 5-mm distance on this map does not reflect the same relationship as a 5-mm distance on a map from a different dataset) [15]. Items 19 ("sensitive to change") and 7 ("important to clinical care") were originally assigned to the "compatible" cluster, but were moved to the "useful" cluster because the investigative team believed that it represented a better conceptual fit. The gray dotted lines within the "useful" cluster and between the "useful" and "compatible" clusters represent how the clusters would have been represented if we had not made this change 


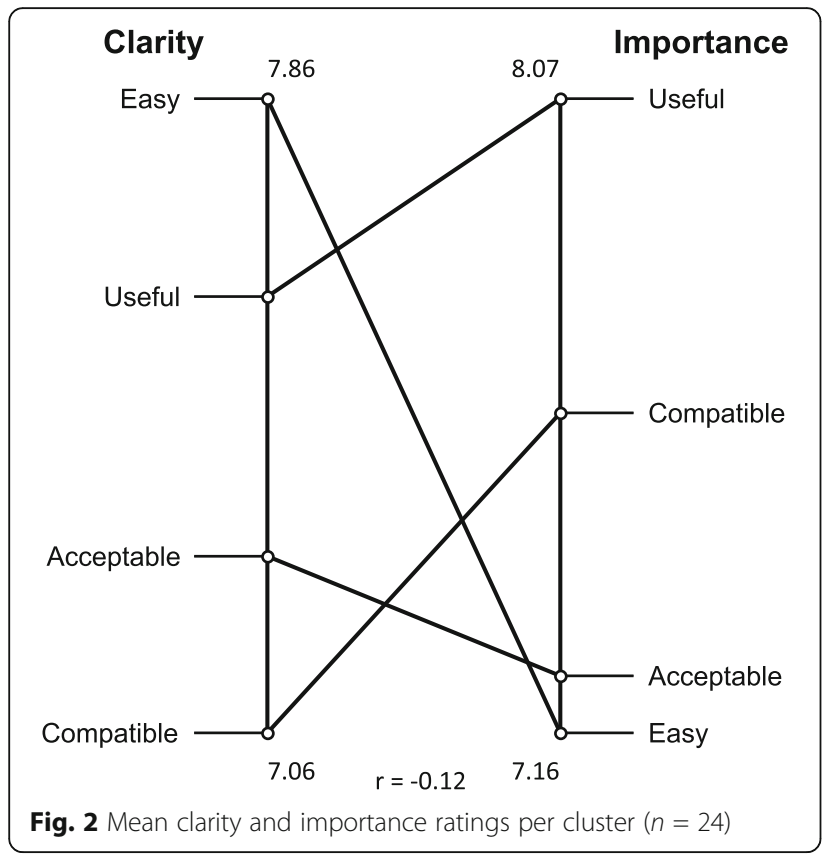

Several limitations should be noted. First, it is possible that engaging our 24 stakeholders in an open process of brainstorming could have yielded a more comprehensive list of potential criteria for the pragmatic construct. Our use of both a systematic literature review and semi-structured interviews with key stakeholders to identify dimensions of the pragmatic construct should largely assuage this concern. Second, our sample primarily included US-based stakeholders working in behavioral health. It is possible that a more diverse group would sort and rate these criteria differently. However, to ensure the relevance of these findings to international stakeholders, we sought input regarding the interpretation and presentation of these findings from our International Advisory Board and learned that the categories and criteria resonated with them. Third, our sample included administrators, clinicians, and researchers; however, it did not include policy makers, who may have rated these criteria differently. Including a larger sample with more diverse stakeholders would have allowed us to examine whether ratings of importance and clarity differed based upon role or work setting, which Aarons et al. [13] have found to be the case in a concept mapping study of stakeholders' perceptions of implementation barriers and facilitators. We believe that these criteria should be generalizable to other contexts, but as they are further developed and applied, it will be important to examine whether they are readily applicable to a diverse array of stakeholders and contexts. Finally, while concept mapping provides a rigorous, mixed methods approach to engaging diverse stakeholders and generating conceptual clarity, there are cases in which the way individual items are grouped does not exactly fit with one's intuitive sense of where they might belong. In some cases when items are located adjacent to a cluster that may provide a better fit, these items can be reassigned as we have done with two of the criteria in this study. In other cases, it is not empirically justified to reassign items. These decisions reflect the judgment of the investigative team and other stakeholders, and others may consider these items differently.

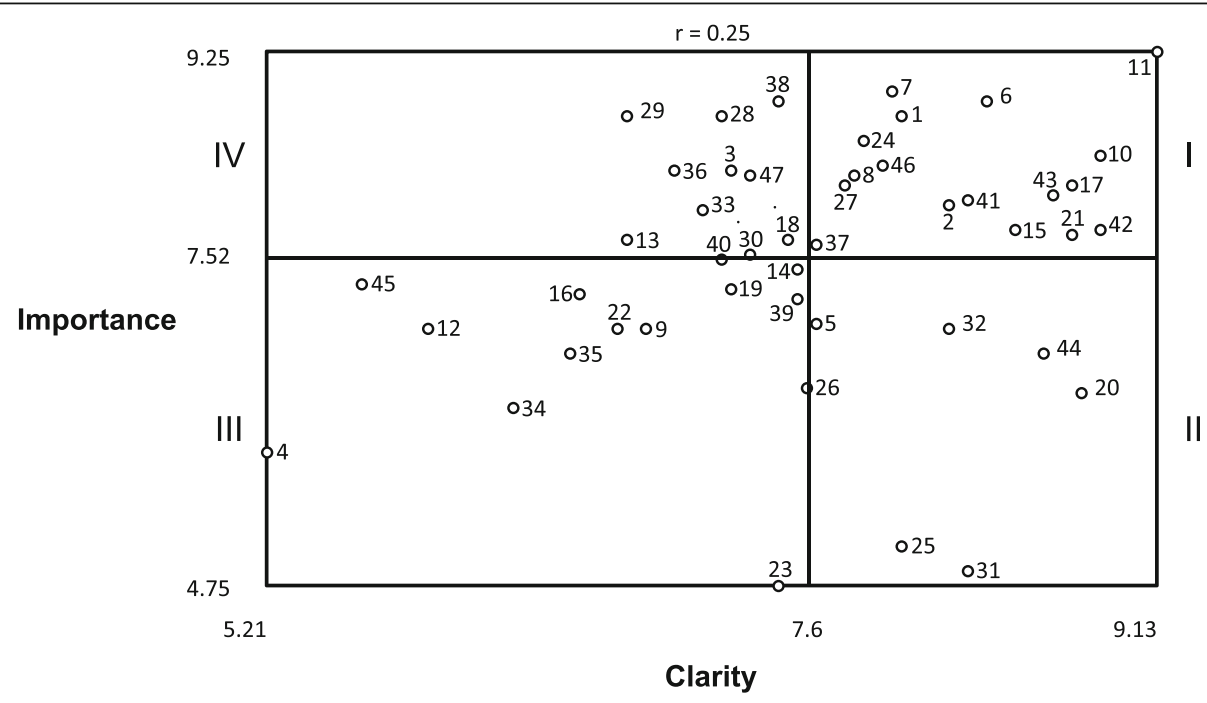

Fig. 3 Go-zone graph of mean clarity and importance ratings $(n=24)$. The range of the $x$ - and $y$-axes reflect the mean values obtained for all 47 of the pragmatic criteria for the clarity and importance rating scales. The plot is divided into quadrants based upon the overall mean values for each rating scale: quadrant I (above the mean for both clarity and importance), quadrant II (above the mean for clarity, below the mean for importance), quadrant III (below the mean for clarity and importance), and quadrant IV (below the mean for clarity, above the mean for importance) 


\section{Conclusions}

This study provides a preliminary list of stakeholderdriven criteria for evaluating the pragmatic qualities of implementation measures. The categories and ratings of these criteria assist in further refinement of the pragmatic construct and facilitate efforts to immediately apply the criteria that appear to be the most clear and important. Ultimately, we hope this nudges the field toward the use of measures that are valid, reliable, and pragmatic.

\section{Acknowledgements}

We would like to thank our International Advisory Board, whose feedback strengthened the manuscript. While some of them have contributed as co-authors (Melanie Barwick, Laura Damschroder, Michel Wensing, and Luke Wolfenden), we would also like to acknowledge Jill Francis, Jeremy Grimshaw, John Ovretveit, Brian Mittman, and Rob Sanson-Fisher.

\section{Funding}

Primary funding for this study was provided by the National Institute of Mental Health (NIMH) through R01MH106510 (Lewis, PI). BJP was also supported by grants and contracts from the NIH, including UL1TR001111, R25MH080916, P30Al050410, L30MH108060, and K01MH113806. MW's position is funded by University Hospital Heidelberg. LW was supported by a NHMRC CDF fellowship and is employed by the Hunter New England Local Health District.

\section{Availability of data and materials}

Data and materials will be made available upon request.

\section{Authors' contributions}

BJP, CFS, HMH, CND, BJW, and CCL conceptualized this study, collected the data, and analyzed the data. MAB, LJD, MW, and LW contributed to the analysis and interpretation of the data. BJP drafted the manuscript. All authors critically reviewed, edited, and approved the final manuscript.

\section{Ethics approval and consent to participate}

The institutional review board at Indiana University, the University of Montana, and the University of North Carolina at Chapel Hill approved all study procedures. Written informed consent was obtained for all study procedures.

\section{Consent for publication}

Not applicable.

\section{Competing interests}

MW is Co-Editor-in-Chief, BW is an Associate Editor, and BJP, LJD, and LW are members of the Editorial Board of Implementation Science. None of the authors were involved in any editorial decisions related to this manuscript. The authors declare that they have no competing interests.

\section{Publisher's Note}

Springer Nature remains neutral with regard to jurisdictional claims in published maps and institutional affiliations.

\footnotetext{
Author details

'Department of Health Policy and Management, Gillings School of Global Public Health, University of North Carolina at Chapel Hill, $1105 \mathrm{C}$ McGavran-Greenberg Hall, 135 Dauer Drive, Campus Box 7411, Chapel Hill, NC 27599, USA. ${ }^{2}$ Hathaway-Sycamores Child and Family Services, Pasadena, CA, USA. ${ }^{3}$ Department of Psychology, University of Montana, Missoula, MT, USA. ${ }^{4}$ Kaiser Permanente Washington Health Research Institute, Seattle, WA, USA. ${ }^{5}$ Department of Global Health and Department of Health Services, University of Washington, Seattle, WA, USA. ${ }^{6}$ Hospital for Sick Children, University of Toronto, Toronto, ON, Canada. ${ }^{7}$ VA Ann Arbor Center for Clinical Management Research and Diabetes QUERI, VA Ann Arbor Healthcare System, Ann Arbor, MI, USA. ${ }^{8}$ Department of General Practice and Health Services Research, University Hospital Heidelberg, Im Neuenheimer Feld, Heidelberg, Germany. ${ }^{9}$ School of Medicine and Public Health, The University of Newcastle, Callaghan, NSW, Australia.
}

Received: 15 May 2017 Accepted: 25 September 2017

Published online: 03 October 2017

\section{References}

1. Proctor EK, Powell BJ, Feely M. Measurement in dissemination and implementation science. In: Beidas RS, Kendall PC, editors. Dissemination and implementation of evidence-based practices in child and adolescent mental health. New York: Oxford University Press; 2014. p. 22-43.

2. Glasgow RE, Riley WT. Pragmatic measures: what they are and why we need them. Am J Prev Med. 2013;45:237-43.

3. Lewis CC, Weiner BJ, Stanick C, Fischer SM. Advancing implementation science through measure development and evaluation: a study protocol. Implement Sci. 2015;10:1-10.

4. Proctor EK, Silmere H, Raghavan R, Hovmand P, Aarons GA, Bunger A, et al. Outcomes for implementation research: conceptual distinctions, measurement challenges, and research agenda. Adm Policy Ment Health Ment Health Serv Res. 2011;38:65-76.

5. Weiner BJ, Lewis CC, Stanick CS, Powell BJ, Dorsey CN, Clary AS, et al. Psychometric assessment of three newly developed implementation outcome measures. Implement Sci. 2017;12:1-12.

6. Stanick CF, Halko H, Dorsey C, Weiner BJ, Powell BJ, Palinkas L, et al. A stakeholder-driven operationalization of the "pragmatic" measures construct. Under Rev.

7. Kane M, Trochim WMK. Concept mapping for planning and evaluation. Thousand Oaks, CA: Sage; 2007.

8. Damschroder LJ, Aron DC, Keith RE, Kirsh SR, Alexander JA, Lowery JC. Fostering implementation of health services research findings into practice: a consolidated framework for advancing implementation science. Implement Sci. 2009;4:1-15.

9. Palinkas LA, Horwitz SM, Green CA, Wisdom JP, Duan N, Hoagwood K. Purposeful sampling for qualitative data collection and analysis in mixed method implementation research. Adm Policy Ment Health Ment Health Serv Res. 2013:42:533-44.

10. Trochim WMK. The reliability of concept mapping. Dallas, Texas; 1993. http://www.socialresearchmethods.net/research/Reliable/reliable.htm.

11. Trochim WMK, Kane M. Concept mapping: an introduction to structured conceptualization in health care. Int J Qual Health Care. 2005;17:187-91.

12. Rosas SR, Kane M. Quality and rigor of the concept mapping methodology: a pooled study analysis. Qual Rigor Concept Mapp Methodol Pool Study Anal. 2012;35:236-45.

13. Aarons GA, Wells RS, Zagursky K, Fettes DL, Palinkas LA. Implementing evidence-based practice in community mental health agencies: a multiple stakeholder analysis. Am J Public Health. 2009;99:2087-95.

14. Lobb R, Pinto AD, Lofters A. Using concept mapping in the knowledge-toaction process to compare stakeholder opinions on barriers to use of cancer screening among South Asians. Implement Sci. 2013;8:1-12.

15. Waltz TJ, Powell BJ, Matthieu MM, Damschroder L, Chinman MJ, Smith JL, et al. Use of concept mapping to characterize relationships among implementation strategies and assess their feasibility and importance: results from the Expert Recommendations for Implementing Change (ERIC) study. Implement Sci. 2015;10:1-8.

16. Tabak RG, Padek MM, Kerner JF, Stange KC, Proctor EK, Dobbins MJ, et al. Dissemination and implementation science training needs: insights from practitioners and researchers. Am J Prev Med. 2017;52:S322-9.

17. Concept Systems, Inc. Concept Systems Global MAX. 2013. http://www. conceptsystems.com/gw/software. Accessed 2017.

18. Hsu C, Sanford BA. The Delphi technique: making sense of consensus. Pract Assess Res Eval. 2007;12:1-8.

19. Lewis CC, Stanick CF, Martinez RG, Weiner BJ, Kim M, Barwick M, et al. The Society for Implementation Research Collaboration Instrument Review Project: a methodology to promote rigorous evaluation. Implement Sci. 2015;10:1-18 